\title{
Statuten der Internationalen Gesellschaft für Geographische Pathologic
}

Die Internationale Gesellschaft für Geographische Pathologie verfolgt den Zweck, die Beziehungen zwischen Krankheiten und geographisch bedingten Faktoren zu studieren.

Die Gesellschaft hat ihren Sitz in der Schweiz.

Mítglieder:

Die Gesellschaft setzt sich aus medizinischen und nichtmedizinischen Per-sönlichkeiten zusammen, die sich für geographisch-pathologische Probleme in-teressieren; sie sind Mitglieder der Gesellschaft. Die Anmeldung als Mitglied hat über das General-Sekretariat zu erfolgen.

Die Mitglieder der Gesellschaft versammeln sich alle 3 Jahre anläßlich einer Konferenz (§ 7); unter Umständen halten sie bei dieser Gelegenheit Vorträge, sie beteiligen sich an den wissenschaftlichen Aussprachen und nehmen mit Stimmrecht an der administrativen Sitzung teil. Jedes Mitglied entrichtet einen Jahresbeitrag von Schweiz. Fr. 5.-; die Beiträge werden entwede $\Gamma$ direkt oder durch Übermittlung des Nationalen Comités an das Generalsekretariat vor dem 1. April des Jahres entrichtet. Die Mítgliederbeiträge sollen vor allem die Kosten der Verwaltung und die Portospesen decken.

4. Nationale und regionale Comités:

In jedem Land oder jeder geographischen Region wird ein nationales, bzw. $\Gamma \beta$ gionales Comité gebildet; dieses besteht aus medizinischen und nichtmedizinischen Persönlichkeiten. Die Zusammensetzung dieser Comités kann sehr wohl wechseln, und zwar je nach dem Thema einer Konferenz. Diese nationalen Comités sind mit den Umfragen und den wissenschaftlichen Untersuchungen betraut, welche von der Gesellschaft bestimmt werden; sie stellen wissenschaft-liche Kommissionen der Gesellschaft dar. Jedes nationale Comité wählt einen PГåsidenten und einen Sekretãr. Der Sekretär ist damit beauftragt: die Verbindung mit dem Generalsekretär aufrechtzuerhalten;

eine vollständige Mitgliederliste für sein Land zu führen, die Jahres-beiträge zu sammeln und sie an den Generalsekretär zu überweisen;

die Ergebnisse der von der Gesellschaft beschlossenen Umfragen in sei-nem Lande zu sammeln und in Form eines oder mehrerer nationaler Berichte verfassen zu lassen. Diese Berichte sind für die Generalbericht-erstatter bestimmt (§ 7).

5. Die Gesellschaft wird von einem leitenden Comité (Executiv-Comité)

verwaltet. Dieses Comité besteht zunächst ex officio aus dem Generalsekretär

und den Präsidenten der beiden vorangegangenen Konferenzen; es umfaßt im

Statuten der Intemationalen Gesellschaft fur Geographische Pathologie 641

weiteren 6 ordentliche Mitglieder und 4 Beisitzer, welche anläßlich der während einer Konferenz abgehaltenen

administrativen Sitzung, durch einfaches Mehr, durch die anwesenden Mitglieder zu wählen sind. Ihr Amt dauert 3 Jahre, bzw. es bleibt bis zur nächsten Konferenz in Kraft und kann erneuert werden.

Einmal gewählt, wird das leitende Comité seinen Präsidenten und zwei Vicepräsidenten selbst ernennen; sie bleiben 3 Jahre im Amt. Der Präsident des leitenden Comités ist Präsident der Gesellschaft und zugleich der designierte Präsident der nächsten Konferenz. Mit dem Generalsekretär vertritt er die Gesellschaft nach außen; er organisiert die Konferenz, der er vorstehen wird, zu-sammen mit dem Generalsekretär. Am Ende derselben erlischt sein Amt alı Präsident.

Das Generalsekretariat ist eine permanente Institution, welche die Be-ziehungen zwischen dem leitenden Comité und den nationalen Comités gewähr-leistet. Der Generalsekretär verwaltet die Geschäfte der Gesellschaft in enger Fühlungnahme mit dem leitenden Comité und besonders mit dessen Präsidenten. Er führt die allgemeine Korrespondenz und unterhält die Verbindungen mit den intemationalen Organisationen, denen die Gesellschaft angehört. Er ist damit beauftragt, aus den verschiedenen Ländern die wissenschaftlichen Un-terlagen zu sammeln, welche den von der Gesellschaft durchgeführten Um-fragen dienen; er organisiert den wissenschaftlichen Teil der Konferenzen in Verbindung mit den lokalen Präsidenten dieser Konferenzen. Mit dem Sekretär der Konferenzen veröffentlicht er die Verhandlungsberichte. Der Generalsekretär ist zugleich Schatzmeister der Gesellschaft. 
In der Regel organisiert die Gesellschaft alle drei Jahre eine Konferenz. Das Diskussionsthema wird, wenn möglich, drei Jahre im voraus bestimmt, und zwar durch die allgemeine Mitgliederversammlung nach Anfrage der Nationalen Comités. Das leitende Comité wählt die Hauptreferenten in Verbindung mit den nationalen Comités. Das Ergebnis der durch die nationalen Comités durchgeführten Umfragen wird in einem nationalen Bericht (oder in $\pi$ lehreren) zusammengefaßt; diese Berichte werden durch die Sekretäre der Nationalen Comités innerhalb genügender Frist dem Generalsekretär zugestellt. Sie bilden die Grundlage der von den gewählten Hauptreferenten anläßlich einer Konferenz vorgetragenen Hauptberichte. Die nationalen Berichte sind geistiges Eigen-tum der nationalen Comités.

Die Konferenzen sind den Mitgliedern der Gesellschaft vorbehalten; Persön-lichkeiten, welche sich für geographischpathologische Probleme oder für das Diskussionsthema interessieren und auf diesem Gebiet selbst arbeiten, können jedoch daran teilnehmen, insofern das nationale Comité ihГes Landes damit einverstanden ist.

8. Jeder Konferenzteilnehmer entrichtet Konferenzgebühren, welche dazu dienen sollen, die Spesen zu decken, welche durch die Vorbereitung und Orga nisation der Konferenz, die Veröffentlichung der Verhandlungen, die Verwaltung des Generalsekretariates und die obligatorischen BeitГäge an die internationalen Organisationen, denen die Gesellschaft angehört, entstehen.

Die Höhe der Konferenzgebühren wird jeweils vom Generalsekretär und 642 Statuten der Internationalen Gesellschaft fur Geographische Pathologie vom Konferenz-Präsidenten je nach den augenblicklichen Erfordernissen und der Devisenlage der verschịedenen Lander festgesetzt.

9. Die Kasse der Gesellschaft wird vom Generalsekretär verwaltet. Während

der administrativen Sitzung, die im Laufe jeder Konferenz stattfindet, werden

zwei Mitglieder als Kassa-Revisoren gewählt. Sie erstatten Bericht über die

Buchführung; auf ihren Vorschlag wird von der Mitgliederversammlung dem

Schatzmeister Entlastung erteilt.

10. Mitgliederversammlung:

Im Verlauf jeder Konferenz versammeln sich die Mitglieder, um über die Geschäfte der Gesellschaft zu beraten. Als besondere Geschäfte sind jeweils vorzusehen: Ernennung des neuen leitenden Comités und Pruning der Kasse; Diskussion und Wahl (durch absolutes Mehr) des Themas für die neue Um-frage sowie Bestimmung des nächsten Tagungsortes. Jedes Mitglied hat das Recht, Anträge eínzureichen, die seiner Meinung nach für die inneren Angelegenheiten der Gesellschaft von Bedeutung sein könnten; solche Anträge sollten mindestens einen Monat vor Konferenzbeginn dem Generalsekretär bekanntgegeben werden.

Mit der praktischen Organisation der Konferenz wird das nationale Comité und besonders dessen Präsident betraut, in dessen Land die Konferenz stattfindet. Dieser Präsident ist für die materielle Organisation der Konferenz verantwortlich. Zusammen mit dem Generalsekretär wird er für die Redaktion, den Druck und Versand der Einladungen und Programme sowie für die Ver-öffentlichungen der Anzeige der Konferenz in der Presse sorgen.

Eine Änderung der vorliegenden Statuten kann vorgenommen werden, nachdem die nationalen Comités mindestens 3 Monate im voraus davon unter-richtet worden sind. Eine Änderung kann nur bei Stimmenmehrheit der Prä-sidenten, der nationalen Comités oder ihrer Vertreter, die anläßlich einer administrativen Sitzung an we send sind, vorgenommen werden. Juli 1957. 